Historic, Archive Document

Do not assume content reflects current scientific knowledge, policies, or practices. 



\title{
DOMOTO BROS.
}

U. 8. Deviartinent of Agriculture.

\author{
Wholesale Price List \\ FOR \\ $1904-1905$
}

Palms, Evergíreens

Ornamental Plants

Roses, Carnations

Chrysanthemums,

Lily Bulbs and

Fruit Trees, Etc.

\section{DOMOTO BROS.}

Japanese Nursery

Central Avenue, near East 14th Street EAST OAKL.AND, CAL. 


\section{Terms:}

All articles are at the risk of the purchaser after being shipped.

A moderate charge will be made to cover cost of material for packing.

Orders from unknown correspondents must be accompanied with the cash. or satisfactory guaranty. C.O.D. orders with at least one-half the amount in cash.

All communications to be addressed to

DOMOTO BROS.

Melrose, Cal. 


\section{DOMOTO BROS.}

\section{Wholesale Price List \\ FOR}

$1904-1905$

Palms, Evergíreens

Ornamental Plants

Roses, Carnations

Chrysanthemums,

Lily Bulbs and

Fruit Trees, Etc.

\section{DOMOTO BROS.}

Japanese Nursery

Central Avenue, near East 14th Street EAST OAHL.AND, CAL.

NURSERY, between Yoakum and Moss Avenues, near

East 14th Street, East Oakland, Cal. 



\section{Palms and Dracæenas}

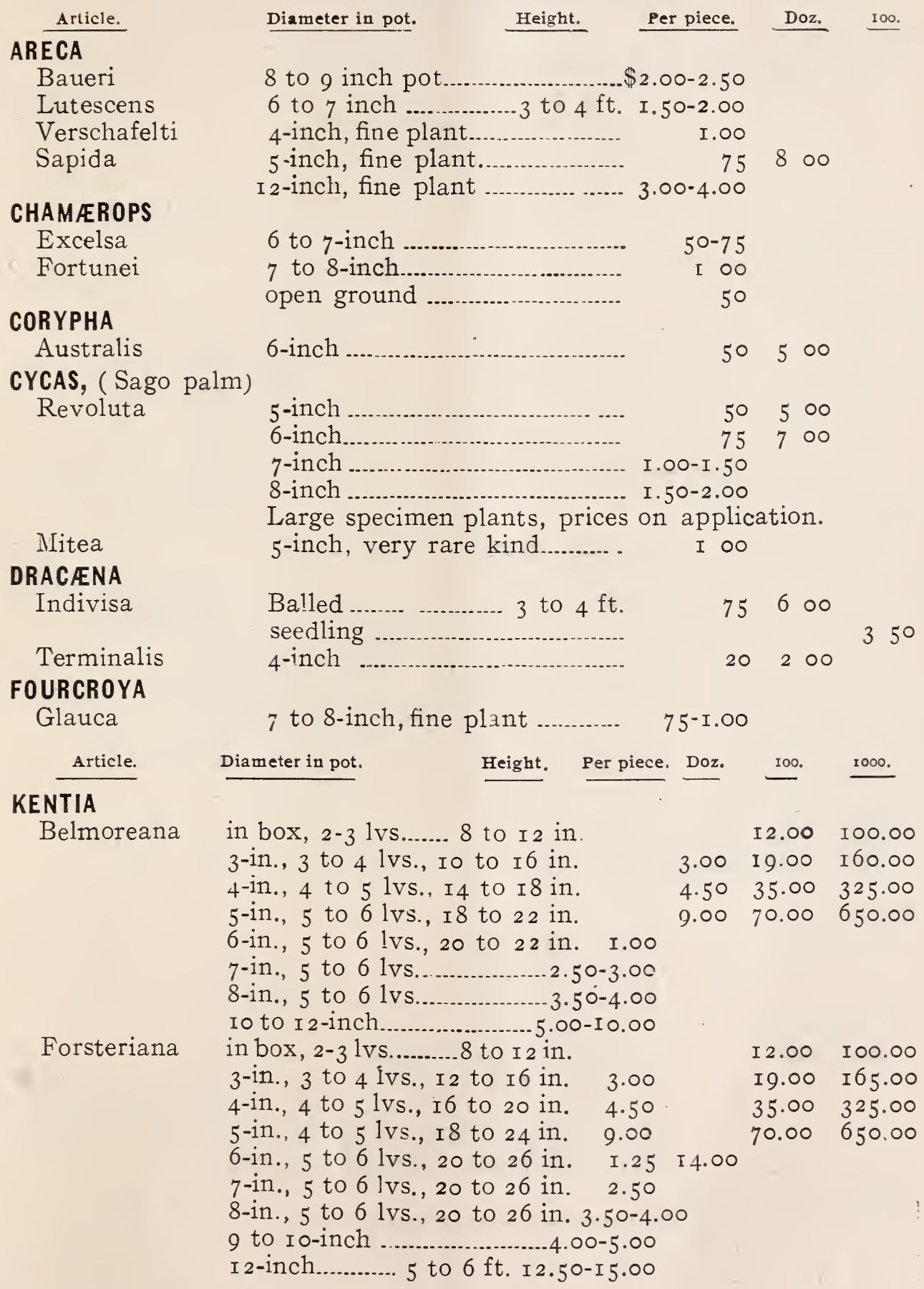


Article. Diameter in pot. Height. Per piece. Doz.

LATANIA

Borbonica

7 to 8 inch

I $50 \quad$ I 500

\section{LIVISTONA}

Rotundifolia seedling, in box

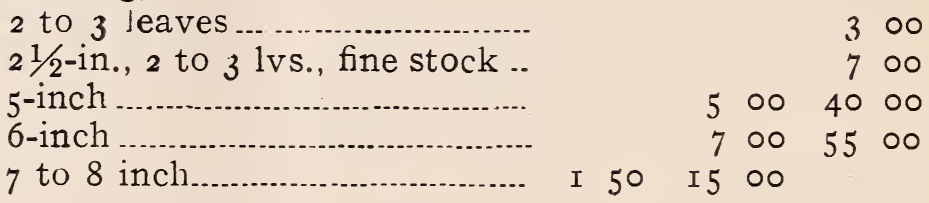

OREODOXA

Regia (Royal palm)

\section{PANDANUS}

Utilis

Veitchi

4-inch

6 to $7 \cdot$ inch

500

5-inch

6-inch

200

7 to ro-inch ........................... 350

Sanderi (golden pandanus)

500

\section{PHENIX}

Canariensis

\section{Reclinata}

Leonancise seedling, in box

$21 / 2$-inch

6 -inch

7-inch,

I 2 -inch.

I 4 X I 4 -inch, in box.

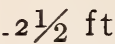

50

I 00 to $31 / 2 \mathrm{ft}$

5 to 6 -inch

8-inch

$2 \mathrm{ft}$

8-inch.

$3.50-4.00$ $50-65$ I 00 IO 00 I 50 I 500

\section{PHORMIUM}

Terax Var egata 6-inch

AloifoliaVariegata 8 to 9 inch

$506 \mathrm{co}$

75900

\section{RHAPIS}

Flabelliformis

6-inch, fine plant 7 -inch, fine plant

8-inch, fine plant.

I. 25 -I.75

I.75-2.50

Variegated

Humilus

5 to 6 -inch, single stock

250

6 to 7 -in., 2 to 3 shoots in pot, 3750 per roo shoots 7 to 8 -in., 4 to 6 shoots in pot, 35 oo per roo shoots 8 to Io-in., 6 to ro shoots in pot, 30 oo per Ioo shoots Specimen plant, prices on application.

\section{YUCCA}

$$
50600
$$




\section{Ferns}

Article

Diameter in pot.

Height.

Per piece. Doz.

100.

ADIANTUM

Cuneatum

Macrophyllum

Gracillimum

Grandiceps

\begin{tabular}{|c|c|}
\hline$\cdots$ & \\
\hline -inch & \\
\hline -inch & \\
\hline inch & 50 \\
\hline Inch & 35 \\
\hline inch & 45 \\
\hline 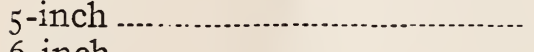 & 35 \\
\hline in & 45 \\
\hline
\end{tabular}

ASPLENIUM NIDUS AVIS

(Bird's Nest Fern) 6-inch

$50 \quad 600$

7 -inch

\section{ALSOPHILA}

Australis, tree fern 6-inch

7-inch

65650

I $\mathrm{OO}$ I $2 \mathrm{OO}$

\section{ASPIDIUM}

Viridescense

6-inch

$35 \quad 400$

\section{CYRTOMIUM}

Falcatum (Holly one of the best hardy ferns Fern)

Fortunei

in house and outdoor

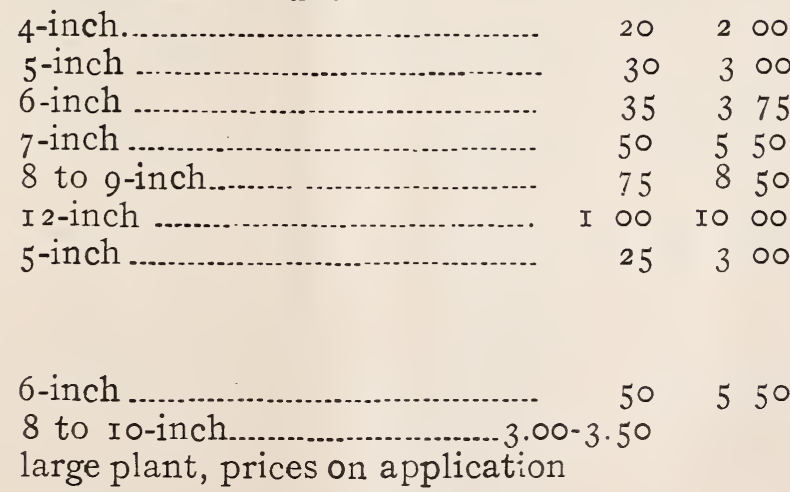

\section{DAVALLIA BULLATA}

(Japan Fern Ball) Hanging Basket

Boat Shape

Wreath Shape

Eagle Shape.

Monkey Shape

Tea House Shape

$\begin{array}{ll}\text { I } 5 & 00 \\ \text { I } 9 & 50 \\ 23 & 00 \\ 39 & 00 \\ 65 & 00 \\ 80 & 00\end{array}$




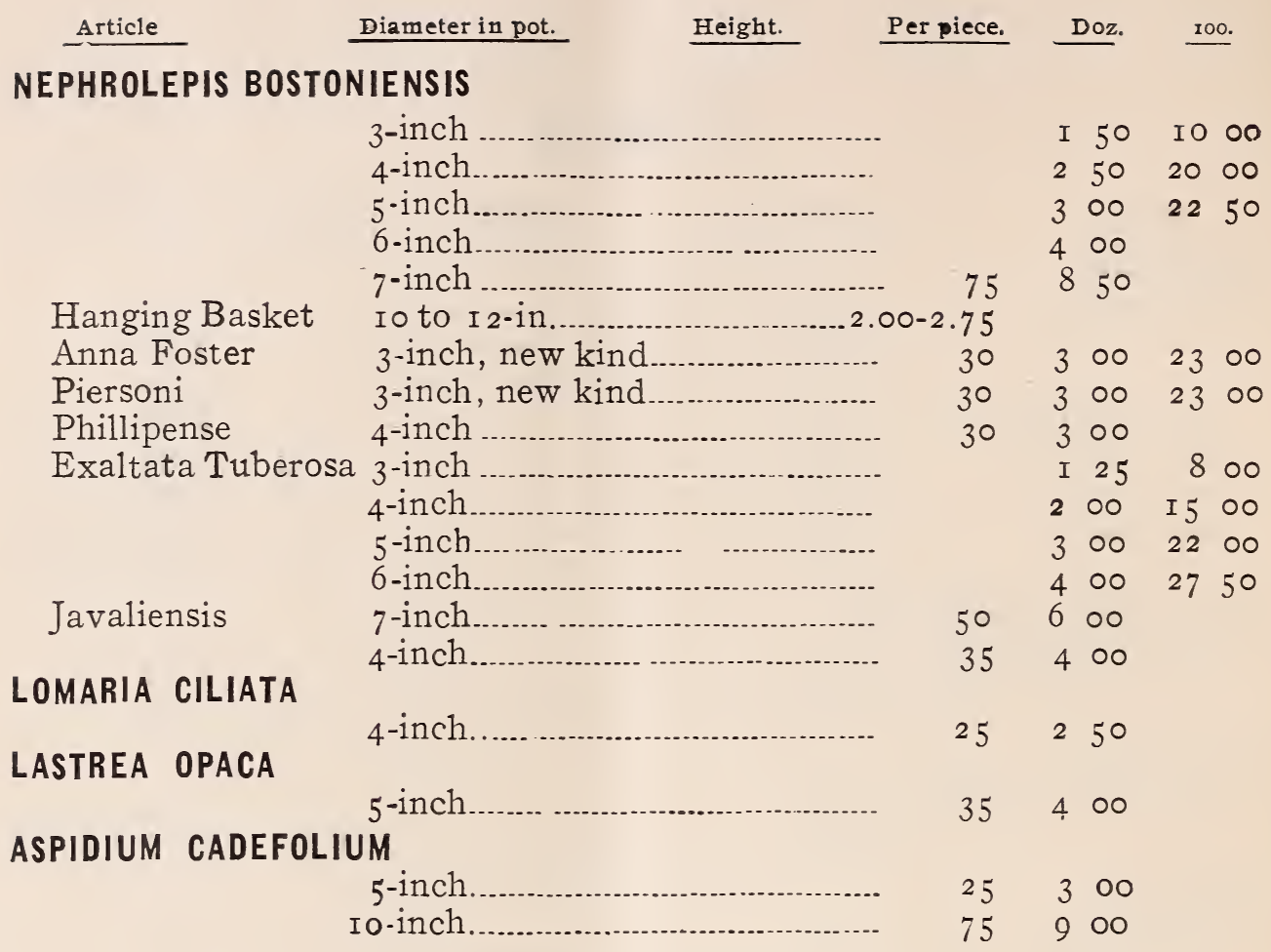

\section{Climbing and Trailing Plants}

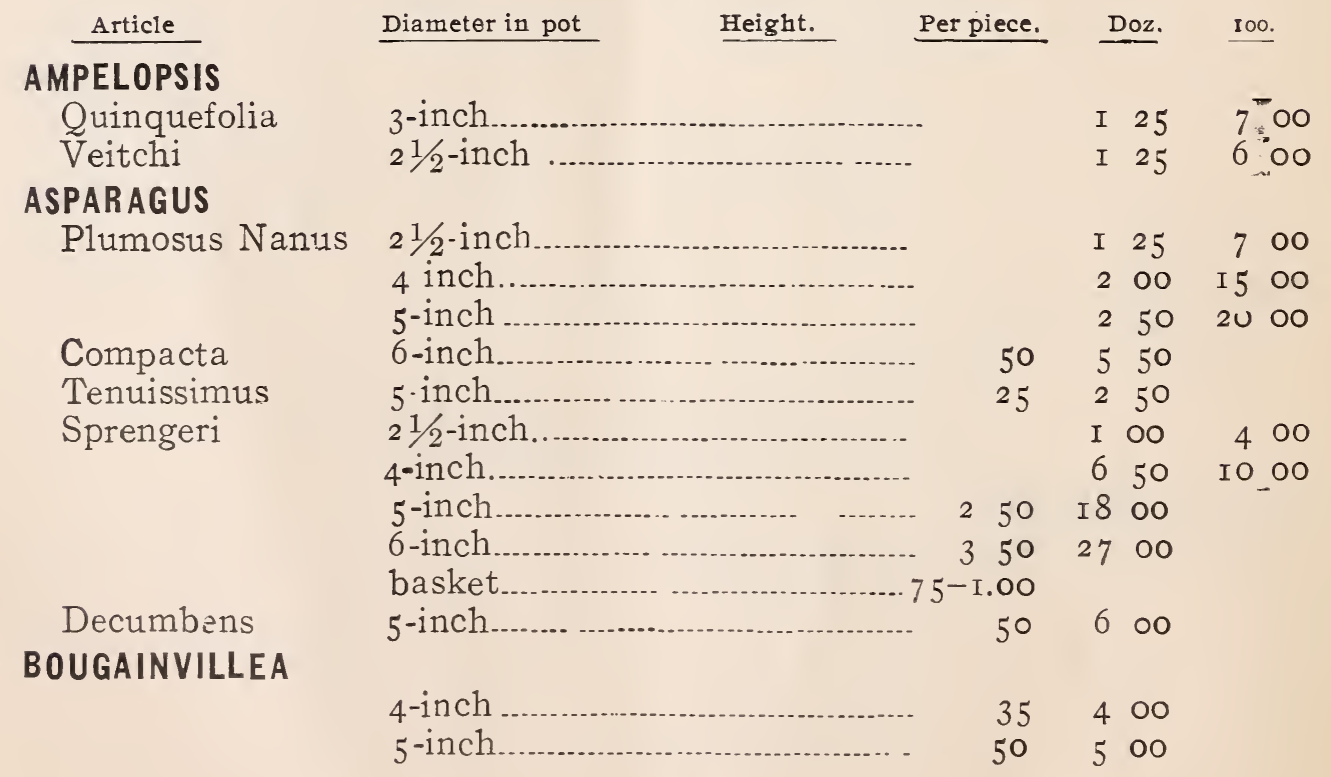


Article

Diameter in pot.

Height.

Per piece.

Doz. $\quad 100$.

\section{CLEMATIS}

Double and single blue, 6 -inch

$35 \quad 400$

Paniculata Grandiflora, 6-inch

35350

\section{FUSCHALY RAPCANSE}

$$
4 \text {-inch. }
$$$$
25250
$$

\section{FUCUS NI PPONICA}

Variegata

\section{HONEYSUCKLE}

Assorted colors

\section{HOYA CARNOSA}

Variegata
5 -inch

6-inch

$21 / 2$-inch

5-inch

25

6-inch, large plant 3 -inch
50

75

I 00

250

35400

I5 I 25

\section{IVY}

English, in varieties 5 to 6 -inch

Japan, variegata one of the best

$25 \quad 250$

35350

JASMINE

Nudiflorum

Revoltum

Ligustrifolium

5 -inch

6 -inch

5 -inch

35350

$50 \quad 500$

$40+50$

\section{MUEHLENBECKIA}

Complexa

4-inch

25300

PASSION VINE

Pink

Scarlet

4 -inch

4-inch.

25200

I 500

SMILAX

21/2-inch

I 00

\section{SWAINSONIA}

Galegifolia Alba

Rubra

5-inch

5 -inch

\section{STEPHANOTIS}

Floribunda

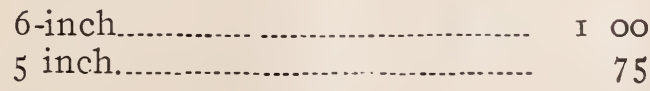

TRUMBEGO

Alba

4-inch

5 -inch

$25 \quad 250$

6 -inch

$30 \quad 300$

Capansise

3 -inch

$50 \quad 600$

I $\mathrm{OO}$ 


\begin{tabular}{|c|c|c|c|c|}
\hline Article. & Diameter in pot. & Per piece. & Doz. & I00. \\
\hline WISTAR IA & & & & \\
\hline Chinesis & 3-in., white and purple, seedling & & & \\
\hline & two years old from graft........... & 35 & 400 & 2800 \\
\hline r & 6-inch, three years old, full & & & \\
\hline & flower buds, for Easter force & 40 & 450 & 3500 \\
\hline & 7 -inch & 75 & 850 & \\
\hline Purple & 7 to 8 -inch ................4 to 5 feet & 75 & 900 & \\
\hline Matifloga & long flowering, 2 yrs. old........... & 35 & 400 & 2800 \\
\hline & pure white, 2 yrs. old................ & 35 & 4. 00 & 2800 \\
\hline & Compacta, 3 yrs. old ................. & 65 & 600 & \\
\hline & double purple, 2 yrs. old.......... & 50 & 550 & \\
\hline & pink, new kind, 2 yrs.old .- & 75 & 900 & \\
\hline & large plant, 4 to 5 yrs. old. & 250 & & \\
\hline Alba Fragrant & latest kind. & I 50 & & \\
\hline
\end{tabular}

\section{Flowering Trees and Shrubs}

Article. $\quad$ Diameter in pot. Height. $\quad$ Per piece. Doz.

\section{ALMOND}

Double, pink and white.

Single, light pink and white

35300

35300

\section{AZALEA}

Indica

Mollis

Japonica

\section{6 -in., in best assorted varieties} 7-inch

7 -inch $2 \mathrm{ft}$.

8-inch $2 \mathrm{ft}$.

$\begin{array}{rrrrrr} & 75 & 8 & 00 & 60 & 00 \\ \text { I } 25 & \text { I } 2 & 50 & 95 & 00 \\ & 75 & 7 & 50 & & \\ \text { I } 25 & \text { I } 3 & 00 & & \end{array}$

\section{CAMELLIA}

Pink, white, red, variegata assortment

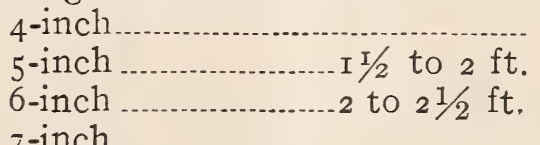

\section{CORONILLA}

7 -inch $50-75$

250 I 8.00

3502500

$450 \quad 3500$

Glauca

Variegata

5-inch

5-inch

$50 \quad 500$

50

\section{CLOTAEGUS CONEPA}

(Japan Thorn)

\section{CLERODENDRON}

Squamatum 
Article.

$\underline{\text { Diameter in pot. }}$

Height. Per piece.

Doz.

I00.

\section{DAPHNE}

Pink and white

$$
\text { 4-inch }
$$

5-inch I 8 in.

ball

FLOWERING CHERRY assorted 20 varieties, 3 to $4 \frac{1}{2} \mathrm{ft}$.

40

450

\section{FUCHSIA}

Assorted

3-inch

IO I OO

\section{GENISTA TINCTORIAL}

(Japan Broom) 4 to 5 -inch .5 to $6 \mathrm{ft}$.

\section{HABESCUS}

Hamabo, golden yellow flower

\section{HYDRANGEA}

In varieties

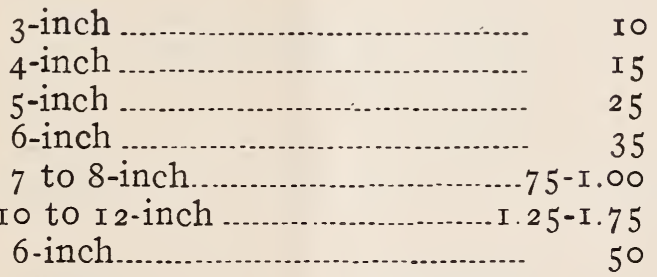

IO I 00

5 I 50

25200

35400

IO $\infty \circ$

I 500

Paniculata

6 -inch

$5 \circ 0$

\section{LAGERSTROEMIA}

Indica, white and red

$50 \quad 500$

\section{MAGNOLIA}

Grandiflora

Conspicua

Obovata

Fuscata

Purpurea

\begin{tabular}{|c|c|}
\hline 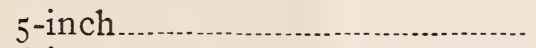 & 35 \\
\hline 5-inch & 5 \\
\hline (n) & 75 \\
\hline ch.... 3 to $31 / 2 \mathrm{ft}$. & \\
\hline 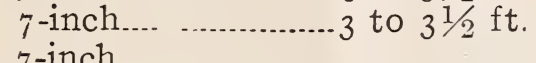 & 75 \\
\hline ch & 5 \\
\hline on & 5 \\
\hline
\end{tabular}

\section{NERIUM}

Oleander

6 in., white and pink, $-3 \mathrm{ft}$.

$50 \quad 500$

\section{PRUNUS}

Mume

30 varieties

$40 \quad 450$

\section{PYRUS}

Spectabilis

\section{PAULOWNIA}

Imperialis

$3 \mathrm{ft}$. 


\section{Miscellanea}

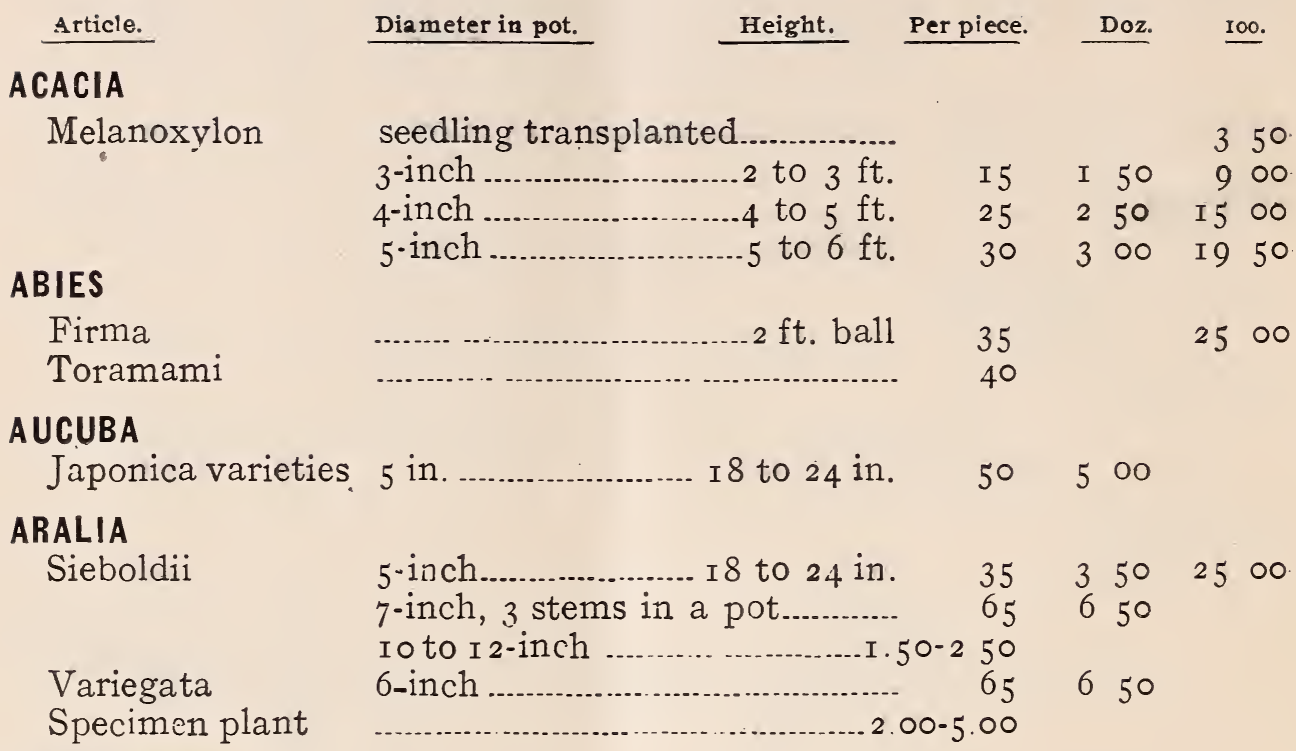

\section{ARAUCARIA}

Excelsa

Bidwellii

Imbricata

$2 \frac{1}{2} \cdot$ inch

6 inch

4 -inch ........................................

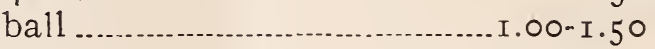

3002000

ANTHERICUM

pot

$2.00-2.50$

Vittatum Variega-

tum

5 -inch

ASPIDISTRA

Lurida

Variegata

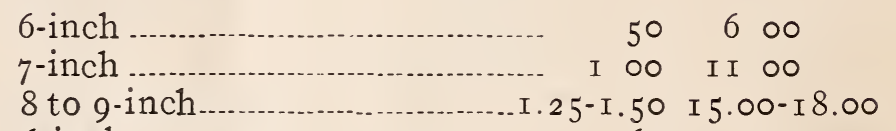

6 -inch

65700

7 -inch

I 25 I 400

8-9-10-inch...........................50-2.00

Also we can furnish per leaf for import stock-

Green, 4.00 per I00, 35.00 per I, 000

Variegata, 7.00 per I00, 65.00 per I,000,600.00 per I0,000

BAMBO0

Varieties 6 to 7 -inch.............................. $50 \quad 500$

\section{BERBER IS}

Japonica

50600

\section{CAREX}

Japonica, variegata 4 to 5 -inch, 3 sorts. 
Article

CAMPHORA TREE

CORYLUS

Helerophilla

\section{CR YPTOMER I A}

(Japan Cedar)

Elegant

\section{COSMOSPORA}

Variegata

DAPHNIPHYLLUM

Glaucescens

Variegata

Macropodum

\section{DEUTZI A}

Lemoineii

Compacta

\section{DENDROPANAX}

Japonica

\section{DIOSMA}

\section{EUONYMUS}

Variegata

\section{ENKI ANTHUS}

Japonica

\section{FARFUGIUM}

Grande

\section{FICUS}

Elastica

Macrophylla

\section{FUNKIA}

Varieties

\section{GARDEN I A}

Grandiflora

Pieno

GINKGO BILOBA

Variegata
Diameter in pot Height Per piece Doz. Ioo.

3 -inch 6 to ro inch

IO

800

4-inch

I 2 to I 8 inch

6-inch

$3 \frac{1}{2}$ to $4 \frac{1}{2} \mathrm{ft}$.

20

$40 \quad 400$

I 500

-3 to $4 \mathrm{ft}$. $40 \quad 400$

40

40

IO I OO

40

75

75

5 -inch

25

5 -inch

25

6-inch 4 to $5 \mathrm{ft}$.

60

5 -inch

$30 \quad 300$

$21 / 2$-inch

IO I 00

fine dwarf tree

I 25

$25 \quad 250$

$50-75 \quad 6.00 \cdot 9.00$

50

25

$50 \quad 600$

$50 \quad 600$

5-inch

7 -inch

$\begin{array}{lll}25 & 250\end{array}$

I 00 I 200 


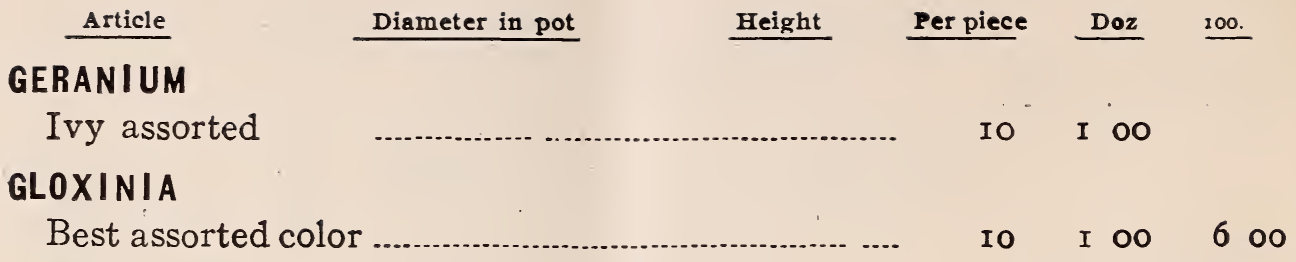

\section{GREVILLEA}

Robusta

8-inch -5 to $7 \mathrm{ft}$.

$75 \quad 700$

\section{HAMAMELIS}

Japonica

50

\section{HEMEROCALLIS}

Flav a

Pleno

\section{HELI OTROPE}

\section{HOLLY}

English

\section{HYPER I CUM}

Mosseana

\section{IRIS}

Kæmpferii

\section{JUNI PERUS}

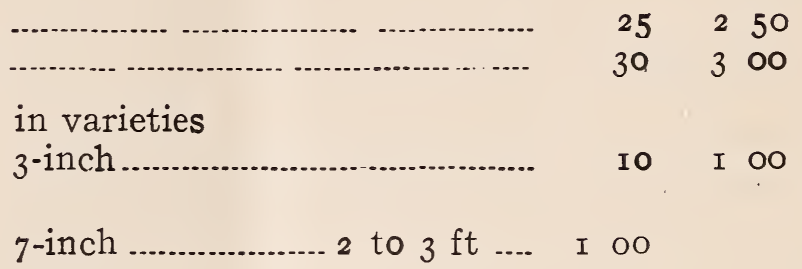

Japonica variegata 6-inch

Chinensis variegata 6 -inch

in 75 varieties.

$\mathrm{New}$ I 2 varieties.

\section{5}

$$
400
$$

2000

Silver

Creeping

6-inch

IO I OO

25300

$35 \quad 400$

$50 \quad 500$

$50 \quad 500$

$35 \quad 400$

\section{KERR I A}

Japonica

$35 \quad 400$

\section{LARIX}

Leptolepis

2 to $3 \mathrm{ft}$....

$30 \quad 300$

\section{LEMON VERBENA}

\section{MUSA}

Ensete

f-inch

$2525^{\circ}$

4-inch

$25 \quad 250$

NANDINA

Domestica

White berry

$$
\text { 5-inch. }
$$

\section{OLEA}

Aquifolium variegata... Fragrant 


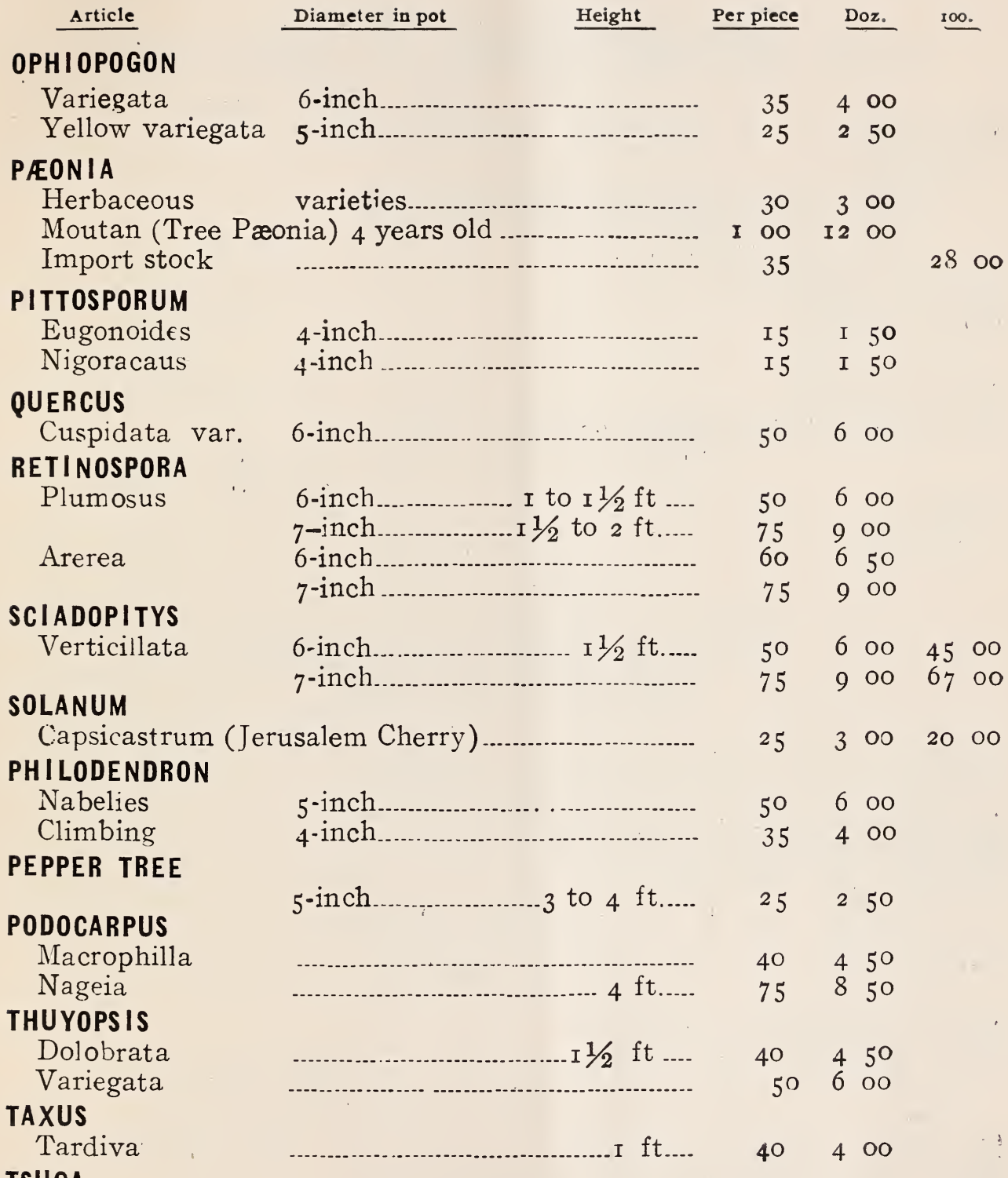

\section{TSUGA}

Sieboldii

\section{THUYA}

S̀quarrosa

Variegata

TORREYA

Nicifera

YEW

Japonic ?

ZANTHOXYLUM

Piperitum
2 to $3 \mathrm{ft} .---5^{-1.00}$

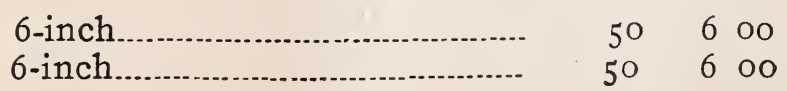

2 to $3 \mathrm{ft} \ldots . .50 \quad 600$

I ft.... $30 \quad 300$

$3 \mathrm{ft} \ldots \quad 35 \quad 400$ 


\section{Japan Fruit Trees}

PERSIMMON

Height. Per piece. Doz. 100. 1000.

Diospyros

Assorted, Hyakume, Gosho Gaki, Dai Dai

$650 \quad 6000$

Maru, Tsurunoko, Hachiya, Yemon,

Tanenashi, Yedoichi, etc.

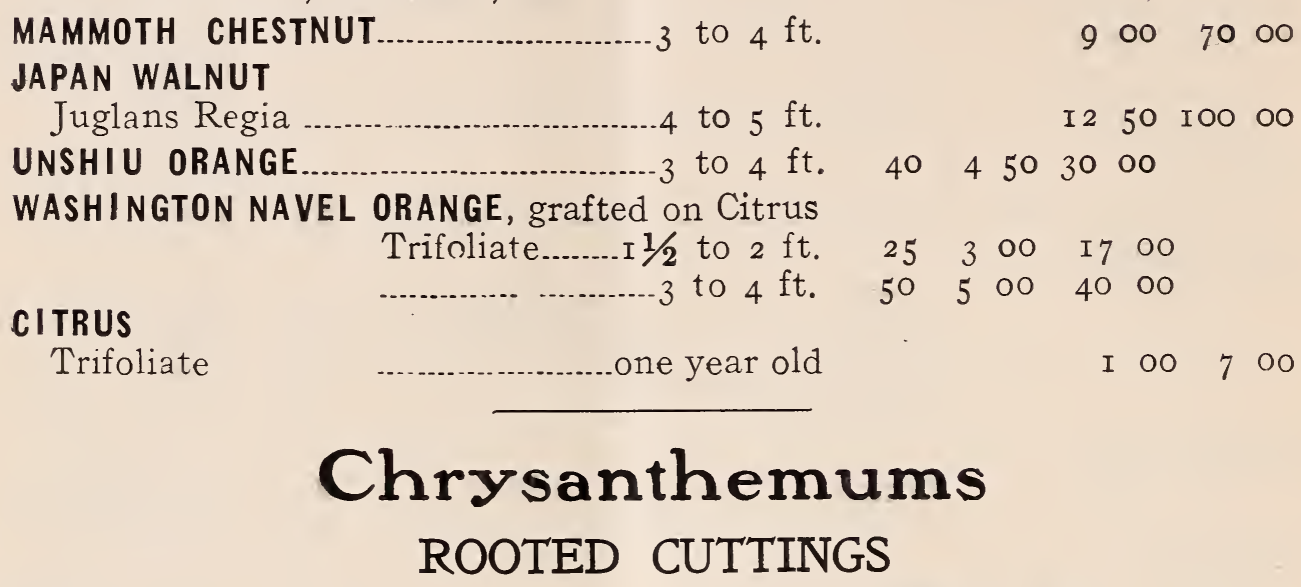

ALGOMA, Japanese white incurved on the Queen

Piece.

Doz.

100

tvpe.

CONVENTION HALL, one of the best white, incurved

COLUMBIA, light pink, large flower

ETHELYN, light rose pink.

F. J. TAGGART, one of the best hairy golden up to date

GLOBOSA ALBA, pure white, incurved of globular form

GOLDEN CHADWICK, large yellow

H. W. BUCKBEE, bright vellow

LORD SALISBURY, yellow, suffused crimson, largest flower

MISS MINNIE BAILEY, clear pink

MDLLE. MARIE LIGER, pearl pink, grandest flower MRS. RUFUS W. SMITH, clear shining white, good for late cut flower

MME. VON ANDRE, yellow, mutual friend

MRS. C. B. FREEMAN, hairy yellow

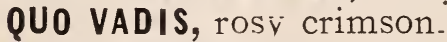

R. E. RICHARDSON, bright, clearest pink.

SOPHIA, bright vellow.

THE YELLOW EATON, bright yellow

JEAN LIABAUD

JUBILEE
\$0 $35 \$ 3 \quad 50$

$25 \quad 250$

$20200 \$ 1000$ 


\section{General Collection of Cut Flower Sorts ROOTED CUTTINGS}

Col. Appieton, Yellow

Major Bonnaffon

White Bonnaffon.

E. Dolleadoze.

Golden Trophy

Glory of the Pacific

J. E. Lager.

Yellow H. J. Jones

White H. J. Jones

Yellow Queen

Polar Queen

Mrs. H. Robinson

Monrovia

Mrs. Hanter, pink

Mrs. E. G. Hill

Mrs. Weeks.

Timot hy Eaton

Ivorv

The Queen

F. Button

Buff Glohe

Golden Gate.

Golden Beauty.

Mrs. O. P. Bassett.

Pennsylvania

Goldmine

Iowa

Princes Chrysanthemum

Mrs. W. B. Chamberlain

Wm. Falconer

Mrs. F. G. Dexter

Louis Boehmer

Cremo

Frank Hardy

Mayflower

Philadelphia

Silver Cloud

White Swan

Adlian

Piece. Doz. I00. I000.

$\begin{array}{llllllllllll} & \$ 0 & 05 & \$ 0 & \$ 3 & 00 & \$ 25 & \end{array}$ 


\section{Carnations ROOTED CUTTINGS}

\section{Enchantress}

Estelle, finest scarlet, $21 / 2$-inch

Flora Hill

Lawson

Iris Miller

Lorna

Louise Bradbury

Portia

Prosperity

Queen Louise

Schwlin

William Scott

White, pink and red of the best assortment on large plant

in box
in box
in box
$21 / 2$-inch
in box
in box
in box
$21 / 2$-inch
in box
in box
of the best assortment on

\section{Roses}

Following varieties ready for delivery Apri] 1st.

$\begin{array}{ll}\text { American Beauty, } & 21 / 2 \text { inch.............. } \\ \text { Bride, } & 21 / 2 \text {-inch............. } \\ \text { Bridesmaid, } & 21 / 2 \text {-inch.............. } \\ \text { Franz Deegen, } & 21 / 2 \text {-inch.............. } \\ \text { Ivory (White Golden Gate), } & 21 / 2 \text {-inch............... } \\ \text { Meteor, } & 21 / 2 \text {-inch.............. }\end{array}$

\section{TWO-YEARS OLD ROSES.}

Caroline Testout

Crimson Rambler

General Jacqueminot.

Kaiserin Augusta Victoria..

Meteor

Ulrich Bruner.
100. 1000 .

$\$ 5 \quad 50 \$ 50 \quad 00$

$\begin{array}{llll}3 & 50 & 30 & 00\end{array}$

$350 \quad 3000$

$\begin{array}{llll}5 & 00 & 45 & 00\end{array}$

$350 \quad 3000$

$\begin{array}{llll}4 & 00 & 35 & 00\end{array}$

\section{EXTRA STRONG ONE-YEAR OLD ROSES.}

Anna de Diesbach.

Cloth of Gold

Banksia (white and yellow)

Cherokee (single and double)

Crimson Rambler.

Papa Gontier.

Golden Gate

La France.

Duchess de Brabant

Lamarque

Ulrich Bruner

Prince Camille de Rohan
Piece. Doz, 100. $\$ 25 \$$ I $50 \$ 1000$
Piece. Doz. Ioo.

\$o I $5 \quad \$$ I $00 \quad \$ 6 \quad 50$ 


\section{NOTICE}

A Special Price will be quoted on import stocks of nursery line and lily bulbs for large orders before the season.

Our lily bulbs are in higher grades than other importers. 


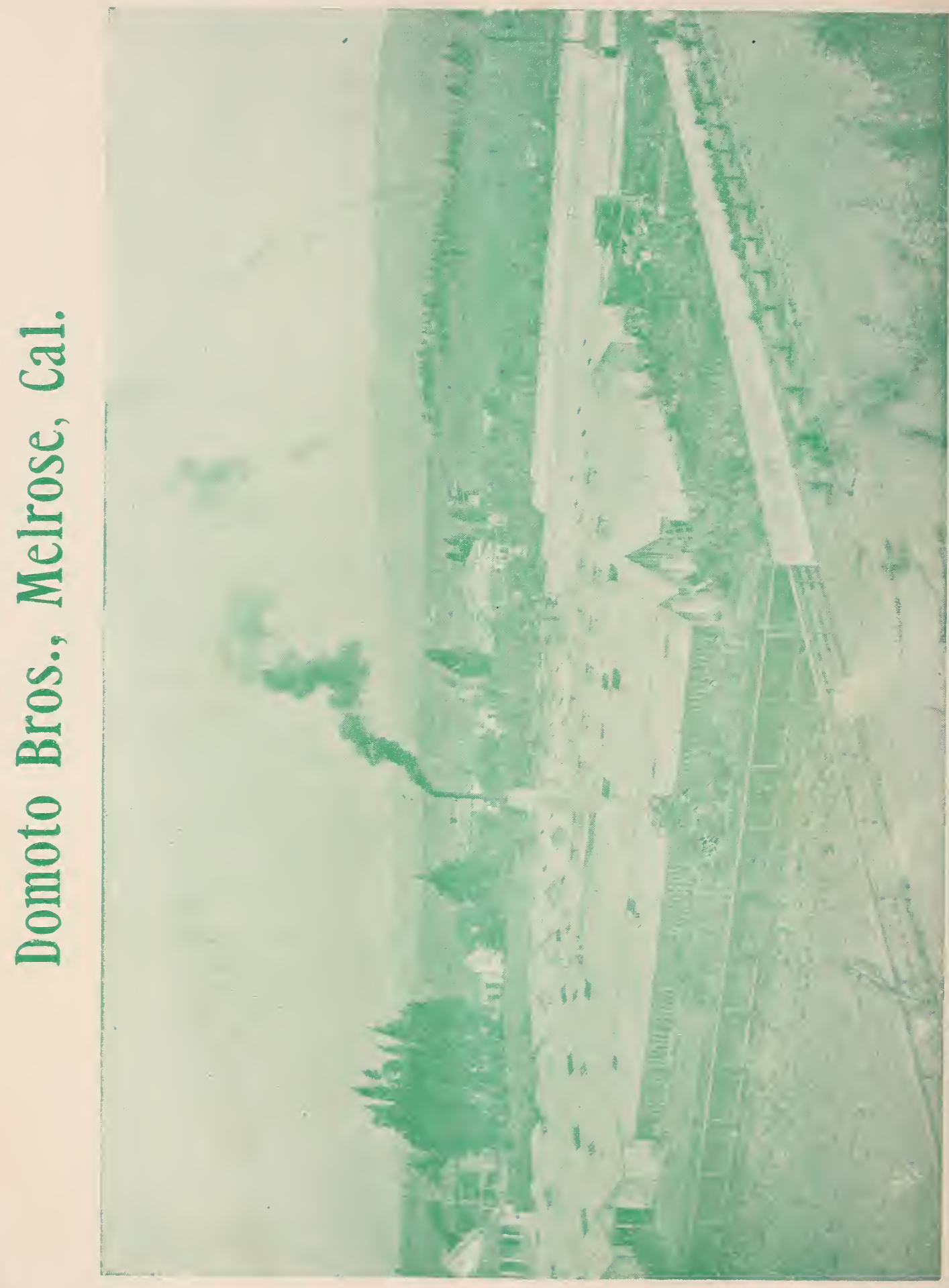

